DOI: https://doi.org/10.46296/yc.v5i8edespen.0090

\title{
RESILIENCIA EN PERSONAS TRANSGÉNEROS FEMENINAS DURANTE CONFINAMIENTO POR COVID-19 EN LA CIUDAD DE PORTOVIEJO
}

\section{RESILIENCE IN FEMALE TRANSGENDER PEOPLE DURING CONFINEMENT BY COVID-19 IN THE CITY OF PORTOVIEJO}

\author{
Sornoza-Macías José Alexander ${ }^{1 *}$; Díaz-Macías Tania Maritza ${ }^{2}$ \\ 1,2 Universidad Técnica de Manabí, UTM. Portoviejo - Ecuador. \\ *Correo: josealex240597@gmail.com
}

\begin{abstract}
Resumen
La resiliencia en personas transgéneros femeninas durante el confinamiento por COVID-19, ha sido una de las problemáticas más fuertes para esta comunidad, la falta de apoyo como ha sido expresado por ellas y a su vez las situaciones que han cursado por las limitantes laborales y las restricciones que se aplicaron para la contención del virus causaron estragos y han sido forzadas a mantenerse entre la población con mayor visibilidad ante la búsqueda de ingresos para la sobrevivencia propia. El objetivo de la investigación es determinar los niveles de resiliencia en personas transgénero femeninas de la Organización Transfemenina de la ciudad de Portoviejo. Para realizar el estudio se aplica una metodología que fue de carácter, mixto cuanti-cualitativo de tipo descriptivo, basado con la técnica de encuesta y entrevista. Para la obtención y análisis de la información se aplicaron instrumentos, los cuales son la escala de resiliencia SV-RES; y entrevista semiestructurada para poder identificar los datos que aporten claridad a la información obtenida. La escala percentil permitió identificar que la resiliencia en las mujeres transgéneros que participaron en la investigación fue de nivel promedio durante la pandemia y estuvieron durante el tiempo de confinamiento con problemas económicos además que la repercusión en sus vidas fue la parte emocional hasta el temor del riesgo ante el contagio por esta enfermedad. Como parte de las conclusiones se indica que las personas transgéneros femeninas deben tener una atención integra sobre los procesos de superación de problemas, y que les brinden un aporte hacia la vida de ellas.
\end{abstract}

Palabras claves: COVID-19, Mujeres transgéneros, Resiliencia, Emergencia sanitaria.

\begin{abstract}
Resilience in female transgender people during confinement due to COVID-19 has been one of the strongest problems for this community, the lack of support as expressed by them and in turn the situations that have occurred due to work limitations and restrictions that were applied to contain the virus caused havoc and have been forced to remain among the population with greater visibility in search of income for their own survival. The objective of the research is to determine the levels of resilience in female transgender people of the Trans Organization of the city of Portoviejo. To carry out the study, a methodology was applied that was of a mixed quantitativequalitative descriptive nature, based on the survey and interview technique. To obtain and analyze the information, instruments were applied, which are the SV-RES resilience scale; and semistructured interview to be able to identify the data that provide clarity to the information obtained. The percentile scale made it possible to identify that the resilience in the transgender women who participated in the research was of an average level during the pandemic and they were during the confinement time with financial problems, as well as that the impact on their lives was the emotional part up to the fear of risk of contagion by this disease. As part of the conclusions, it is indicated that transgender women must have comprehensive attention to the processes of overcoming problems, and that they provide them with a contribution towards their lives.
\end{abstract}

Keywords: COVID-19, Transgender women, Resilience, Health emergency.

Información del manuscrito:

Fecha de recepción: 16 de noviembre de 2020

Fecha de aceptación: 15 de enero de 2021.

Fecha de publicación: 29 de enero de 2021. 


\section{Introducción}

La organización mundial de salud, en su informe sobre "El género y la COVID-19" indico que las pandemias $y$ los brotes tienen efectos diferenciales en las mujeres y los hombres. Desde el riesgo de exposición y la sensibilidad biológica a la infección hasta las consecuencias sociales y económicas, es probable que las experiencias de las personas varíen según sus características biológicas y de género y su interacción con otros determinantes sociales.

En este sentido, las características que se mantienen como parte de su proceso integro en mujeres $y$ hombres, logra establecer una relación entre los efectos unipersonales, y que a su vez reproducen particularidades únicas que son integras desde su propio ser interno y a la vez en su forma con el contexto que lo rodea. De acuerdo con esto la emergencia al brote de la COVID-19, también significa que las mujeres llegan a formar parte de la categoría de mayor denigración, esto se puede ver afectado desde el enfoque de género como aproximación a las formas de replicación de un ejercicio patriarca, que en ciertos aspectos predominan comportamientos y prácticas agresivas por el confinamiento.

El 31 de diciembre de 2019, la Comisión Municipal de Salud y Sanidad de Wuhan (provincia de Hubei, China) informó sobre un grupo de 27 casos de neumonía de etiología desconocida, con una exposición común a un mercado mayorista de marisco, pescado y animales vivos en la ciudad de Wuhan, incluyendo siete casos graves. El día 11 de marzo, la OMS declaró la pandemia mundial. Desde el inicio hasta la fecha de este informe se han alcanzado más de 50 millones de casos notificados en todo el mundo. (Secretaria de Estado de Sanidad, 2020).

De acuerdo con la Organización Mundial de la Salud (OMS), el coronavirus es una extensa familia de virus que pueden causar enfermedades tanto en animales como en humanos. En los humanos, se sabe que varios coronavirus causan infecciones respiratorias que pueden ir desde el resfriado común hasta enfermedades más graves como el síndrome respiratorio de 
Oriente Medio (MERS) y el síndrome respiratorio agudo severo (SRAS). El coronavirus que se ha descubierto más recientemente causa la enfermedad por coronavirus COVID19.

El primer caso en la Región de las Américas se confirmó en Estados Unidos el 20 de enero del 2020, y Brasil notificó el primer caso en América Latina y el Caribe el 26 de febrero del 2020. Esto lo reafirma Organización Panamericana de la Salud en el 2020, (OPS).

La llegada "tardía" del virus a Latinoamérica en comparación con Asia y Europa, ha abierto una ventana de oportunidad que ha permitido a la mayoría de los países tomar medidas enérgicas tempranas tratando de frenar el avance de la pandemia (declaración de "estado de emergencia sanitaria" o "estado de excepción por catástrofe", promoción de medidas de higiene más rigurosas, búsqueda y contacto de casos sospechosos, restricción de la movilidad y aglomeraciones (Beylis, 2020).

La crisis de COVID-19 no solo está afectando al nivel del empleo, sino también a su composición que se encuentra reflejada da desde los diferentes sectores económicos que se han visto afectados por cuarentenas y confinamientos a distintos niveles (Beylis, 2020, et, at.). Esto sin dejar afuera los factores y replicas sobre las masas humanas, y a su vez sobre el factor de primera instancia, sobre qué pasa con las poblaciones que son afectadas por la llegada de este virus.

Siendo así, el impacto del COVID-19 en las sociedades, gobiernos y economías de la región pueda llegar a ser distinto en comparación a otras regiones del mundo, debido a que presentan limitaciones existentes en sus sistemas públicos y escases de recursos (Lafuente, 2020). Para entender esta problemática de la expansión del virus en Latinoamérica se recomienda analizar tres ámbitos distintos que se interrelacionen entre sí, esto es: Lo político, el económico y el sanitario (Peñafiel, 2020). Que logra afectar a las personas que se encuentran en cada uno de sus territorios.

Luego de este acontecimiento las alertas sobre el COVID se desarrollaron de manera 
exponencial, el estado de esta emergencia logro expandirse en cada país de las diferentes regiones americanas. Esto también es un objeto de indagaciones al sistema de gobiernos; por esta misma situación cualquier modelo predictivo, es que puede ser la acción de los gobiernos que están llevando a cabo sobre la base de procesos de prueba y error. En algunos casos la crisis sanitaria está adquiriendo fuerza en crisis políticas, como se ve en la contradicción frecuente entre autoridades locales y gobiernos centrales o entre países en el seno de bloques de integración regional (CEPAL, 2020).

Por otra parte, el objeto de esta variable de estudio es lograr y comprender que pasa con las poblaciones que se denominan como "Población clave", a niveles, sociodemográficos, culturales y sobre autoconstrucción política. En este sentido, las mujeres transgéneras que forman parte de esta población llegan a ser una de las principales identidades de género, con mayor número de discriminación que se encuentra emergido en los factores: laboral, social, económico y político.
Torres (2020). En medios de comunicación y en las redes sociales se han denunciado episodios sistemáticos de abuso de poder policial autorizados por una retórica que en ciertos sectores ha ganado consenso de "linchamiento social" de quienes no cumplen las normas. Ello es especialmente peligroso en América Latina, donde la presencia de gobiernos autoritarios y conservadores es caldo de cultivo fértil para la normalización del acoso social y la represión (pág. 8).

De acuerdo con la mencionado con anterioridad los sistemas que permiten visibilizar la realidad de las poblaciones claves, son las mismas organizaciones que cumplen un roll de importancia total "para las personas que no tienen voz", que, dentro de su propio contexto, en este caso las personas transgéneros 0 reconocidas también como mujeres trans, desarrollan una estructura de identidad, que constituyen unas de poblaciones más invisibles dentro de la sociedad. Cuando se habla de estas personas, la parte medica siempre lo señala como una ser que tiene condiciones diferentes, y que deben estar preparados los profesionales para prestar una 
atención que esté sujeta a los estudios de esta población, deben contener conocimientos y haber realizados, procesos y estudios avanzados en la formación de una psicología especializada, en donde logre reconocer síntomas que se desarrollan durante el proceso de transición en estas personas (Zapata, 2019).

Entonces, el autor Hernández (2017). Determina que se encuentra comprendida a la identidad como un constructo o producto de lo relacional, esta deja de verse como algo individual, estable e inmodificable. Una misma persona puede transformar su identidad indefinidamente, variarla según los diferentes contextos, ser una cosa y ser otra a la vez, incluso aunque estas no parezcan coherentes.

Un dato muy importante, es que las poblaciones vulnerables tienden a desarrollar problemáticas, que se encuentran emergidas como la comunicación entre las siglas, esto quiere decir "GLBT", consta de varias identidades y prácticas, pero que a su vez desarrollan individualismo por sus características únicas (...) Dentro de la misma comunidad GLBTI, se puede indicar que hay niveles de violencia en las mismas personas de la comunidad, que están dentro los sujetos con identidades de género y orientaciones sexuales diferentes de lo que es cisgéneros 0 heterosexuales (Ribeiro, 2019).

Entonces, sobre esta situación es circunstancial identificar, si también dentro de las siglas "GLBTI", se desarrolla una estructura de discriminación, que puede ser que las mujeres trans, tienen una doble discriminación, en primera instancia por su género y segundo por mismos integrantes de los grupos vulnerables, entonces Barrientos (2019). Responde desde una manera conceptual de que la mayor visibilidad e interés por estudios sobre las personas trans, en Latinoamérica puede ser porque no existen revisiones en español actualizadas de la literatura científica internacional sobre aspectos relativos a la salud de las personas trans.

La mujer transgénero como dentro de la concepción del auto concepto se llega a reconocer con su propia identidad en ciertos casos también esta construcción se encuentra ligadas al aspecto bilógico y puede 
repercudir sobre cómo se construyen desde el aspecto político, social y de la formación de sus talentes físicos hasta los mentales.

Para aquello, y con procedentes de violencia y de falta de apoyo, en una mujer trans, el análisis de la resiliencia se reconoce también como la capacidad de hacer frente 0 superar la adversidad, lo que puede relacionarse directamente con el proceso que un individuo experimenta en la búsqueda de su identidad sexual. Pueden ser estos mismos factores los que actúen como variables precursoras y que facilitan al proceso de cambio frente a las diferentes dificultades que se puedan presentar (Cole, 2018, p.10).

Pero será posible, que este aspecto mantenga una característica, que sea única para la población de mujeres trans, 0 que sea un comienzo que ayude a sistematizar nuevos conceptos, sobre las identidades y las resistencias que estos tienen, para llevar a cabo sus procesos de transición. Sin embargo, se abre un abanico que es capaz de establecer como ha sido la llega del COVID-19 en las mujeres trans (...). La autora Calderón (2020), en su texto de redacción, hace hincapié en que La pandemia por COVID-19 expone la realidad a la que se enfrentan las mujeres transgéneros que la falta de reconocimiento de su identidad de género y derechos por parte del Estado, violencia y discriminación. La pandemia también aumenta la crudeza de esa realidad porque impide migrar, algo que para algunas de ellas es la única posibilidad de sobrevivir.

El impacto de la pandemia del COVID-19 en las mujeres trans privadas de la libertad ha sido desproporcionado comparado con otros grupos, en particular, por las diversas interseccionalidades que atraviesan sus vidas. Ante esta preocupante situación, organizaciones de la sociedad civil lideradas $y / 0$ que trabajan con personas trans privadas de la libertad han desarrollado diversas iniciativas para garantizar el ejercicio pleno de la dignidad y la protección de los derechos humanos de este colectivo (García, 2020).

De acuerdo con el contexto de Ecuador de disponer a los Gobiernos Autónomos Descentralizados Municipales, dentro del marco de sus competencias, emitan y aprueben una Resolución u Ordenanza 
Municipal que regule el uso de mascarillas. En la misma se normará el uso obligatorio de mascarillas quirúrgicas a nivel comunitario a fin de reducir la transmisión del virus, y, se restringirá: 1) el uso de las mascarillas tipo respirador $\mathrm{N}-95$ a nivel comunitario; y, 2) la libre circulación de las personas que hayan sido diagnosticados por COVID-19, recordando la obligación de guardar el aislamiento, hasta cumplir con su periodo de recuperación.

Esta situación social y de emergente forma de visualizar, se permite que se dé una comprensión de la formación de un proyecto de vida partiendo de los significados que permean desde sus sistemas y las configuraciones de su experiencia personal, en donde se tienen en cuenta las posibilidades y recursos, necesidades, objetivos, orientaciones y aspiraciones; cada uno de estos enmarcado dentro de un contexto social que debe ser considerado en las relaciones y en la dinámica en la que se vea inmerso. (Pérez, 2018).

\section{Objetivo general}

Determinar los niveles de resiliencia en personas transgénero femeninas de la Organización Transfemenina de Portoviejo.

\section{Metodología}

La investigación se la realizo mediante el método mixto cuanticualitativo de tipo descriptivo, basado en la técnica de encuesta y entrevista. Para la obtención y análisis de la información se aplicaron instrumentos, los cuales fueron la escala de resiliencia SVRES; conformada por 60 ítems divididos en 12 factores, estos midieron niveles generales y áreas específicas de resiliencia. Una entrevista semiestructurada; con el objetivo de identificar los datos más precisos que aporten claridad a la información obtenida en la investigación. Estos instrumentos fueron aplicados de manera online a través de videollamadas por la aplicación de WhatsApp y Zoom y con el diseño de un formulario de Google drive, además que pueden estuvieron sujetos a cambios de acuerdo con la crisis sanitaria que atraviesa el país por la presencia del coronavirus (COVID-19). 


\section{Resultados}

\subsection{Análisis e interpretación de resultados obtenidos}

Nivel sociodemográfico de las participantes en la investigaciónmétodo de aplicación entrevista estructurada.

Tabla 1. Nivel sociodemográfico.

\begin{tabular}{|c|c|c|c|}
\hline Edad & & & \\
\hline Ítems & Cantidad & $\%$ & Total \\
\hline $18-20$ & 0 & $0 \%$ & 0 \\
\hline $20-30$ & 2 & $25 \%$ & 2 \\
\hline $30-40$ & 5 & $62 \%$ & 5 \\
\hline $40-50$ & 1 & $13 \%$ & 1 \\
\hline & & & 8 \\
\hline Nivel de escolaridad & & & \\
\hline Ítems & Cantidad & $\%$ & Total \\
\hline Primaria & 0 & 0 & 0 \\
\hline Secundaria & 5 & $62 \%$ & 5 \\
\hline Educación superior & 3 & $38 \%$ & 3 \\
\hline Profesional & 0 & 0 & 0 \\
\hline Ocupación laboral & & & 8 \\
\hline Ítems & & & \\
\hline Estilista & Cantidad & $\%$ & Total \\
\hline Trabajo sexual & 1 & $12 \%$ & 1 \\
\hline Servicios comunitarios & 2 & $25 \%$ & 2 \\
\hline & 5 & $63 \%$ & 5 \\
\hline & & & 8 \\
\hline
\end{tabular}

Fuente: Autores de la investigación, (2020).

\section{Análisis e interpretación:}

En la tabla 1, se puede identificar que el $62 \%$ de población investigada tienen edades entre los 30-40 años, siendo una población adulta y que puede establecer los factores $y$ situaciones por la que cursan en su vida personal y social.

El nivel de escolaridad de la población de estudio, es muy importante resaltarlo, porque se puede reconocer los avances de las mujeres transgéneros en el área de educación y cuáles son los aproximaciones educativas que ellas tienen, el $62 \%$ de las participantes tienen nivel de educación medio, entre estos (Bachillerato), mientras que el 38\% tienen nivel de educación superior, en este sentido el asumir que varias mujeres transgénero cuenta con estudios de educación ayuda a comprender que existe un mayor alcance en la concepción de superior personal. 
El $63 \%$ de las participantes realizan servicios comunitarios, con las diferentes organizaciones que cumplen un roll vitalicio para atender la demanda de atención primaria, en caso de VIH (SIDA), violencia de genero e integración para la seguridad del ejercicio sexual, como es parte de la exploración de la investigación el 25\% de la muestra tomada, indico que realiza servicio sexual como parte de sus ocupaciones laborales, entonces se puede indicar que los servicios que brindan las organizaciones de atención prioritaria, son incidentes ante la atención de la población que realiza este tipo de actividad laboral.

Tabla 2. Resiliencia en COVID-19.

\begin{tabular}{|c|c|c|c|}
\hline \multicolumn{4}{|l|}{ Pregunta $1-¿$ ¿Conoce usted sobre la resiliencia? } \\
\hline Ítems & Cantidad & $\%$ & Total \\
\hline Si & 3 & $37 \%$ & 3 \\
\hline \multirow[t]{2}{*}{ No } & 5 & $63 \%$ & 5 \\
\hline & & & 8 \\
\hline \multicolumn{4}{|l|}{$\begin{array}{l}\text { Pregunta 2- Considerando que la resiliencia es la } \\
\text { capacidad de afrontar la adversidad. ¿Cree usted ser } \\
\text { resilente? }\end{array}$} \\
\hline $\mathrm{Si}$ & 6 & $75 \%$ & 6 \\
\hline \multirow{2}{*}{ No } & 2 & $25 \%$ & 2 \\
\hline & & & 8 \\
\hline \multicolumn{4}{|l|}{ Pregunta 3- ¿En qué grado afectó la pandemia su vida? } \\
\hline Mucho & 7 & $87 \%$ & 7 \\
\hline Algo & 1 & $13 \%$ & 1 \\
\hline \multirow{2}{*}{ Poco } & 0 & 0 & 0 \\
\hline & & & 8 \\
\hline \multicolumn{4}{|l|}{$\begin{array}{c}\text { Pregunta 4- ¿Durante la cuarentena tuvo alguna } \\
\text { pérdida de un familiar, amigo, conocido, alguien o algo } \\
\text { de mucha importancia para usted? }\end{array}$} \\
\hline $\mathrm{Si}$ & 4 & $50 \%$ & 4 \\
\hline \multirow[t]{2}{*}{ No } & 4 & $50 \%$ & 4 \\
\hline & & & 8 \\
\hline \multicolumn{4}{|l|}{$\begin{array}{l}\text { Pregunta 5- ¿Llegó a sentir o pensar que estuviera } \\
\text { enfermo o se podría enfermar en todo este tiempo? }\end{array}$} \\
\hline $\mathrm{Si}$ & 6 & $75 \%$ & 6 \\
\hline \multirow[t]{2}{*}{ No } & 2 & $25 \%$ & 2 \\
\hline & & & 6 \\
\hline
\end{tabular}

Fuente: Autores de la investigación, (2020) 


\section{Análisis e interpretación:}

En la tabla 2, se pudo identificar ciertos factores que se tomaron en cuenta sobre la presencia de problemáticas provocadas por la pandemia y que a su vez lograron causar un nivel de deshabilitación emocional en las mujeres transgéneros.

En la pregunta 1.- sobre el conocimiento de resiliencia, las mujeres transgéneros el $63 \%$ de la población entrevista indico que no tienen conocimiento sobre que es la resiliencia, mientras un $37 \%$ respondió que si tenían conocimiento, y utilizaron termino como, la forma de solucionar sus problemas; "forma de aprender a sobrevivir", pero según la Real academia española de la lengua (RAE ), la resiliencia es la capacidad de adaptación de un ser vivo frente a un agente perturbador $\mathrm{o}$ un estado $\mathrm{o}$ situaciones adversas. Entonces la falta de conocimiento sobre esta área de las ciencias humanas puede llegar a ser un factor, para que exista una intervención sobre cómo se puede superar los problemas mediante la búsqueda de soluciones $y$ acciones afirmativas.
En la pregunta 2.- Esta pregunta asume la consideración sobre si las mujeres transgéneros tienen consideración sobre que es la capacidad de resiliencia el $75 \%$ respondió que sí, porque esta interrogante especificaba la "capacidad de afrontar adversidades", entonces bajo esta categoría ellas asumieron que, si tienen resiliencia, pese a que desconocen el significado de este término. Para la autora Solano (2020). Existen situaciones que preceden la concepción de la resiliencia, así mismo por la variación que es los antecedes expuestos y que es importante mencionar que una de las ciencias que se encarga de brindar apoyo y bienestar es la resiliencia como parte integradora del ser humano.

En correlación de la pregunta 3.- La pandemia causo, muchos estragos a nivel mundial, pero, sin embargo, los contextos sociales son diferentes y entre ello las formas sociales que involucran su composición. Las mujeres transgéneros forman parte de estos contextos también se vieron afectadas, y dentro de la ciudad de Portoviejo, la población estuvo bajo la misma orden de restricción a nivel 
mundial y bajo los protocolos de movilidad que se establecieron. Por una parte, el $87 \%$ de ellas indicaron que fueron afectadas mucho por la situación, sufriendo varias problemáticas que las hacen vulnerables, desde el enfoque discriminativo, de roll y género y sobre la incidencia ante la falta de recursos económicos, aspectos que son relevantes dentro de la población. Un dato importante sobre esta situación es que, pese a las medidas de prevención sobre el virus, los aumentos de la tasa de femicidios/feminicidios en cuarentena por parte de parejas y exparejas. Puede darse también, que algunas de las mujeres transgéneros estuvieron cursando algún tipo de situación, este análisis también promueve la forma que el confinamiento puede causar estragos por su forma tan repentina y a su vez aumentando índices en otros contextos como la violencia misma de género.

La pérdida de un familiar, o de algún ser que forme parte de la muestra de estudio se encuentra representada en la sección cuatro, fue un factor de las formas de auto superación por la pérdida de las vidas de algún ser que conformase parte de ellas. En este aspecto el $50 \%$ de ellas afirmaron haber perdido alguien, mientras el otro $50 \%$ indico que no han tenido perdida de algún familiar.

La pregunta 5.- Indicaba si las mujeres transgéneros llegaron a sentir algún tipo de síntomas del COVID-19. El 75\%, respondió que llegaron a sentir síntomas, mientras el $25 \%$ indico que no sintieron síntomas.

Tabla 3. Escala percentil.

\begin{tabular}{|l|c|c|}
\hline \multicolumn{3}{|c|}{ INTERPRETACION DEL PUNTAJE PERCENTIL } \\
\hline PUNTAJE PERCEPTIL & $0-25$ & BAJO \\
\hline PUNTAJE PERCEPTIL & $26-74$ & PROMEDIO \\
\hline PUNTAJE PERCEPTIL & $75-99$ & ALTO \\
\hline
\end{tabular}

Autor: Teste SRVS. 
Tabla. 4. Análisis de los resultados de la prueba SV-RES.

\begin{tabular}{|c|c|c|c|c|}
\hline $\begin{array}{c}\text { PUNTAJE } \\
\text { DIRECTO }\end{array}$ & $\begin{array}{c}\text { PUNTAJE EN VALOR } \\
\text { PERCENTIL }\end{array}$ & FRECUENCIA & PORCENTAJE & \\
\hline 194 & $\begin{array}{c}\text { PUNTAJE PERCENTIL } \\
0-25=\text { BAJO }\end{array}$ & 1 & BAJO & $25 \%$ \\
\hline $243-261$ & $\begin{array}{c}\text { PUNTAJE PERCENTIL } \\
26-74=\text { PROMEDIO }\end{array}$ & 3 & PROMEDIO & $75 \%$ \\
\hline 0 & $\begin{array}{c}\text { PUNTAJE PERCENTIL } \\
75-99=\text { ALTO }\end{array}$ & 0 & & $0 \%$ \\
\hline & TOTAL & 4 & & \\
\hline
\end{tabular}

Autor: Autores de la investigación, (2020).

Figura 1. Análisis de los resultados de la prueba SV-RES.

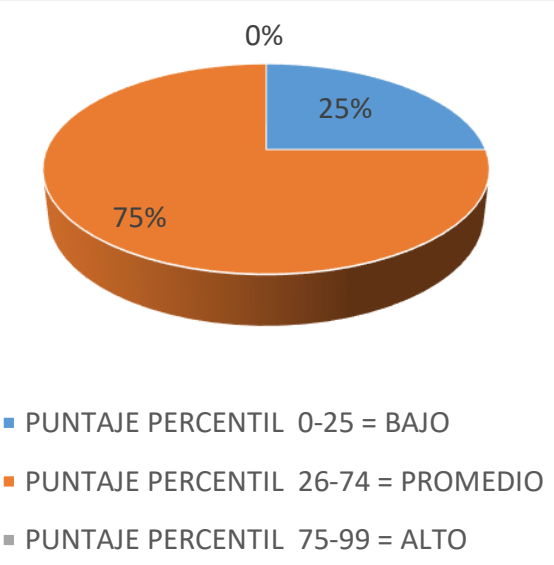

Análisis e interpretación:

En la tabla 4, sobre la escala percentil, se pudo identificar que la resiliencia en las mujeres transgéneros que participaron en la investigación y estuvieron durante el tiempo de confinamiento por COVID
-19 , fue de manera promedio, lo cual puede indicar que la resiliencia en ellas se encuentra desarrollado a raíz de los factores que han cursado en su vida, y que la autoprotección de esta, ante la situación presentada era el salvaguardar su vida.

Tabla 5. Yo soy - Yo estoy.

\begin{tabular}{|c|c|c|c|}
\hline \multicolumn{4}{|c|}{ YO SOY - YO ESTOY } \\
\hline F1 IDENTIDAD & F2 AUTONOMIA & F3 SATISFACCION & F4 PRAGMATISMO \\
\hline 3 & 3 & 3 & 4 \\
\hline $23 \%$ & $23 \%$ & $23 \%$ & $31 \%$ \\
\hline
\end{tabular}

Autor: Autores de la investigación, (2020). 
Figura 2. Yo soy - Yo estoy.

\section{Análisis e interpretación:}

En la tabla 5, el nivel más elevado que tuvieron en respuesta al Test aplicado, se reconoció en el pragmatismo con un $31 \%$, lo cual puede indicar que de acuerdo a los referentes teóricos dentro del área de estudio indican que las mujeres transgéneros son víctimas de violencia y de no aceptación por la sociedad, el primer nivel de resiliencia que es "Yo soy - Yo estoy", definiría en este caso al pragmatismo que las características y patrones que desarrollan las mujeres transgénero bajo el confinamiento, es que temían pos el contagio, y que sufriendo la perdida de familiares, que les han hecho reivindicar y accionar de manera más fuerte su presencia como un roll de visibilidad ante la emergencia por COVID-19.

Tabla 6. Yo tengo.

\begin{tabular}{|c|c|c|c|}
\hline \multicolumn{4}{|c|}{ YO TENGO } \\
\hline F5 VINCULOS & F6 REDES & F7 MODELOS & F8 METAS \\
\hline 4 & 4 & 4 & 4 \\
\hline $25 \%$ & $25 \%$ & $25 \%$ & $25 \%$ \\
\hline
\end{tabular}

Autor: Autores de la investigación, (2020).

Figura 3. Yo tengo.

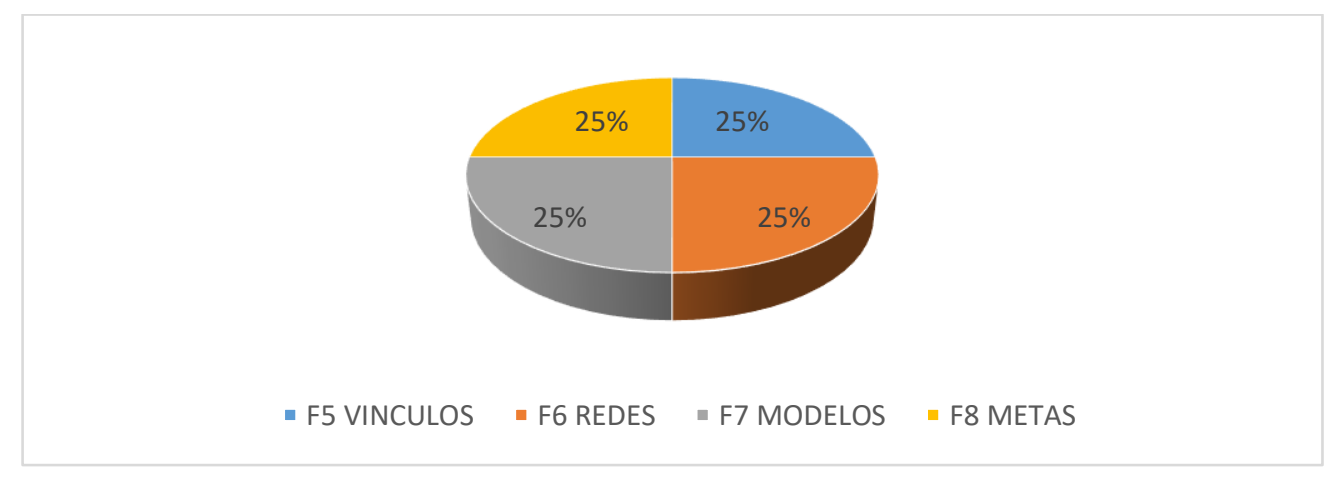




\section{Análisis e interpretación:}

El 25\% de las mujeres transgéneros, indicaron que tenían vínculos, que les permitían desarrollar un grado de confianza, y que a través de estos lograron mantener una estabilidad emocional frente a la pandemia por COVID-19

El $25 \%$ también sostuvo que mantuvieron una parte de comunicación con las redes de apoyo, y que les permitieron proveer alimentos y ayudas humanitarias por parte de la ONG, que se encuentran liderando las estrategias de $\mathrm{VIH}$, dentro de la ciudad de Portoviejo. Esto se contrasta al que nivel de resiliencia "Yo tengo", es que las mujeres transgéneros de la ciudad pueden tener un apoyo que viene desde organismos particulares, que les ayudo prevalecer la existencia y la seguridad conforme a sus necesidades presentadas por COVID-19.

Las siguientes respuestas que formulan el $50 \%$ de los porcentajes de la investigación, también proyectaron que tienen modelos de superación y que en parte la atención brindada por las redes de apoyo le ayudaron a superar el confinamiento de manera más positiva, y así mismo las metas planteadas por esta situación, son parte de los diferentes problemas que han cursado por la poca presencia del ejercicio laboral y así mismo; la equivalencia que tienen para proponerse el subsistir durante el tiempo que la pandemia continúe dentro de la ciudad.

Tabla 7. Yo puedo.

\begin{tabular}{|c|c|c|c|}
\hline \multicolumn{4}{|c|}{ YO PUEDO } \\
\hline F9 AFECTIVIDAD & F10 AUTOEFICACIA & F11 APRENDIZAJE & F12 GENERATIVIDAD \\
\hline 4 & 4 & 4 & 3 \\
\hline $27 \%$ & $27 \%$ & $27 \%$ & $20 \%$ \\
\hline
\end{tabular}

Autor: Autores de la investigación, (2020).

Figura 4. Yo puedo.

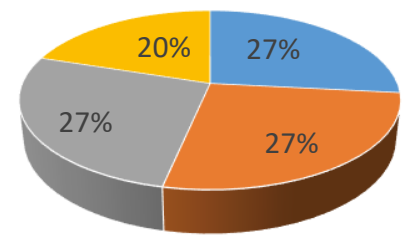




\section{Análisis e interpretación:}

Dentro del análisis de la tabla 7 , es prescindible establecer la relación que existe entre: Afectividad, Autoeficacia, Aprendizaje, y es que las mujeres transgéneros, tiene ciertas variables que fueron presentadas ante la necesidad del afecto y apoyo familiar, $y$ en el caso de esta investigación que se planteó por confinamiento del COVID-19 logra entender que las mujeres transgéneros tienen cierta carencia de afectividad y que esta sujetamente vinculada con la autoeficacia que exploran actitudes que son poco positivas para ellas.

\section{Conclusiones}

La resiliencia en tiempo de COVID19 en personas transgéneros ha demostrado que ha sido un factor predominante para el doble grado de discriminación y sobre los efectos no positivos para las vidas de ellas, por una parte la pandemia revelo que las personas transgéneros sufren problemáticas que van desde lo personal hasta lo social, y que están vinculadas a la falta de atención por ser grupos que no llegan a ser visibles por sus realidades. En otro aspecto los problemas sobre ellas son producto de antecedentes que han causado secuelas en su forma interior, y que si bien es cierto se encuentra fulgurado en medidas regulares para la superación de problemas.

\section{Bibliografía}

Álvarez, P, Harris, P. (2020). COVID19 en América Latina: Retos y oportunidades. Rev. chil. pediatr. 91(2). 179-182. Disponible en: https://scielo.conicyt.cl/scielo. php?script=sci_arttext\&pid=S 0370$41062020000200179 \& \operatorname{lng}=\mathrm{es}$

http://dx.doi.org/10.32641/rch ped.vi91i2.2157.

Barrientos, J, Saiz, J, Gómez, F, Espinoza, R, Cárdenas, Bahamondes, J. (2019). La Investigación Psicosocial Actual Referida a la Salud Mental de las Personas Transgénero: Una Mirada Desde Chile. PSYKHE, 28 (2). 1-13, ISSN: 0717-0297. Recuperado de https://scielo.conicyt.cl/pdf/ps ykhe/v28n2/0718-2228psykhe-28-02-

psykhe_28_2_1482.pdf

Beylis, Guillermo, Roberto Fattal Jaef, Michael Morris, Ashwini Rekha Sebastian y Rishabh Sinha. 2020. Efecto viral: COVID-19 y la transformación 
acelerada del empleo en América Latina y el Caribe. Estudios del Banco Mundial sobre América Latina y el Caribe. Washington, DC: Banco Mundial. doi:10.1596/978-1- 46481638-3. Recuperado de https://openknowledge.worldb ank.org/bitstream/handle/109 86/34413/211448SP.pdf

Calderón, C. (2020). [Mensaje de un blog]. Encerradas y sin derechos: así viven la pandemia las mujeres trans en Honduras. Recuperado de https://contracorriente.red/20 20/07/07/encerradas-y-sinderechos-asi-viven-lapandemia-las-mujeres-transen-honduras/

CEPAL. (2020). América Latina y el Caribe ante la pandemia del COVID-19 Efectos económicos y sociales. Recuperado de https://repositorio.cepal.org/bi tstream/handle/11362/45337/ 4/S2000264 es.pdf

Cole, S, Irarrazábal, J, Quinteros, P. (2018). La transexualidad en Chile y sus factores resilentes. (Tesis de pregrado). UNIVERSIDAD GABRIELA MISTRAL. Santiago, Chile. Recuperado de

http://repositorio.ugm.cl/bitstr eam/handle/20.500.12743/18 24/CD\%20ME.PSI\%20\%285 \%29\%202018.pdf?sequence

$=1 \&$ isAllowed $=\mathrm{y}$

García, T. (2020). [Mensaje de un blog]. Mujeres trans tras las rejas: estrategias de resistencia en la pandemia del COVID-19. Recuperado de https://www.wola.org/es/anali sis/mujeres-transencarcelamiento-covid-19/

Hernández, M. (2017). Resiliencia y comunidad LGTB+ ¿Tener derechos es suficiente? (Tesis de grado). Universidad de la Laguna. Recuperado de https://riull.ull.es/xmlui/bitstre am/handle/915/5592/Resilien cia\%20y\%20comunidad\%20L GTB\%2B\%20\%C2\%BFTener $\% 20$ derechos\%20es\%20sufic iente.pdf?sequence $=1$ \&isAllo wed $=\mathrm{y}$

Mejías, M, González, R, Rodríguez, M. (2016). LA RESILIENCIA EN JÓVENES TRANS COMO FACTOR PROTECTOR DE LA DISCRIMINACIÓN SOCIAL. Recuperado de http://webcache.googleuserc ontent.com/search?q=cache:i c3wpF0dNusJ:www.cibaman z2020.sld.cu/index.php/cibam anz/cibamanz2020/paper/do wnload/168/110+\&cd=28\&hl= es $-419 \& \mathrm{ct}=\mathrm{clnk} \& \mathrm{gl}=\mathrm{ec}$

Organización mundial de la salud. (2020). El género y la COVID 19. Recuperado de https://apps.who.int/iris/bitstre am/handle/10665/332242/W HO-2019-nCoV- 
Advocacy_brief-Gender2020.1-spa.pdf

Organización Mundial de la Salud. (2020). Preguntas y respuestas sobre la enfermedad por coronavirus (COVID-19). Recuperado de https://www.who.int/es/emerg encies/diseases/novelcoronavirus-2019/

Organización Panamericana de Salud. (2020). Informes de situación de la COVID-19. Recuperado de https://www.paho.org/es/infor mes-situacion-covid-19

Pérez, H, Peña, D, Silva, S. (2018). Procesos resilientes en hombres y mujeres que pertenecen a la etapa del ciclo vital de la juventud que atravesaron una ruptura amorosa. (Tesis de Pregrado). Universidad Santo Tomas. Bogotá, Colombia. Recuperado de https://repository.usta.edu.co/ bitstream/handle/11634/1357 4/2018danielapena.pdf?sequ ence $=1$ \&isAllowed $=y$

Ribeiro, L, Neves, S, Antunes, M. (2019). Representaciones sociales de personas transgénero travestis $y$ transexuales) sobre la violencia. Revista de psicología, 37 (2). 495-527, ISSN: 0254-9247. Recuperado de http://www.scielo.org.pe/pdf/p sico/v37n2/a07v37n2.pdf
Secretaria de Estado de Sanidad. (2020). Enfermedad por coronavirus, COVID-19. Recuperado de https://www.mscbs.gob.es/pr ofesionales/saludPublica/cca yes/alertasActual/nCov/docu mentos/ITCoronavirus.pdf

Solano, M, Mera, R. (2020). REDES DE APOYO Y RESILENCIA EN MUJERES TRANSGÉNEROS: CASO CIUDAD DE BOLÍVAR, ECUADOR. Revista Científica Multidisciplinaria Arbitrada YACHASUN. 4, (7). ISSN: 2697-3456. Recuperado de http://webcache.googleuserc ontent.com/search?q=cache:

gahkv4H8WkJ:www.editorialib kn.com/index.php/Yachasun/ article/download/56/128+\&cd $=2 \& \mathrm{hl}=\mathrm{es}-419 \& \mathrm{ct}=\mathrm{clnk} \& \mathrm{gl}=\mathrm{ec}$

Torres, A. (2020). LA SEGURIDAD DE LAS MUJERES FRENTE A LA COVID-19: NUDOS CRÍTICOS PARA AMÉRICA LATINA. Recuperado de: http://library.fes.de/pdffiles/bueros/laseguridad/16137.pdf

Zapata, A, Díaz, K, Ahumada, L, Sales, L, Linares, J, Zapata, F. (2019). Atención de salud de personas transgéneros para médicos no especialistas en Chile. Rev Med Chile, 147. 65-72. 